\title{
COSMIC RAY ACCELERATION AT RELATIVISTIC SHOCKS
}

\author{
M. Ostrowski \\ Obserwatorium Astronomiczne, Uniwersytet Jagielloniski, \\ 171 Orla Str., 30-244 Kraków, Poland
}

(Received July 31, 2002)

\begin{abstract}
Theoretical studies of cosmic ray particle acceleration in the first-order Fermi process at relativistic shocks are reviewed. At the beginning we discuss the acceleration processes acting at mildly relativistic shock waves. An essential role of oblique field configurations and field perturbations in forming the particle energy spectrum and changing the acceleration time scale is discussed. Then, we report on attempts to consider particle acceleration at ultra-relativistic shocks, often yielding an asymptotic spectral index $\sigma \approx 2.2$ at large shock Lorentz factors. We explain why this result is limited to the cases of highly turbulent conditions near shocks. We conclude that our present knowledge of the acceleration processes acting at relativistic shocks is insufficient to allow for realistic modelling of the real shocks. The present review is a modified extended and updated version of [M. Ostrowski, in Frontier Objects in Astrophysics and Particle Physics (Vulcano Workshop), edited by F. Giovannelli, G. Mannocchi (1999), p. 319.]
\end{abstract}

Key words: cosmic rays, relativistic shock waves, gamma ray bursts.

PACS number(s): 95.30.Qd, 95.85.Pw, 98.70.Rz, 98.70.Sa

\section{INTRODUCTION}

Relativistic plasma flows are detected or postulated to exist in a number of astrophysical objects, ranging from

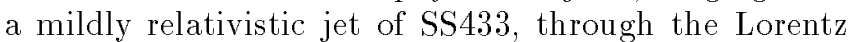
factor of a few jets in AGNs and galactic 'mini-quasars', up to ultra-relativistic outflows in sources of gamma ray bursts and, possibly, in pulsar winds. As nearly all such objects are efficient emitters of synchrotron radiation and/or high energy photons requiring the existence of energetic particles, our attempts to understand the processes generating cosmic ray particles are essential for understanding the fascinating phenomena observed. Below we will discuss the work carried out in order to understand the cosmic ray first-order Fermi acceleration processes acting at relativistic shocks. One should note that in the present discussion we consider the high energy particles with gyroradii (or mean free paths) much larger than the shock thickness defined by the compressed 'thermal' plasma. The present review is an updated version of [1], also including an extended discussion of the acceleration processes acting at ultra-relativistic shocks [2].

\section{PARTICLE ACCELERATION AT NON-RELATIVISTIC SHOCK WAVES}

Processes of the first-order particle acceleration at non-relativistic shock waves were widely discussed by a number of authors during the last two decades (for review, see, e.g. [3-6]). Below, we review the basic physical picture and some important results obtained within this theory for test particles, to be later compared with the results obtained for relativistic shocks.

The simple description of the acceleration process preferred by us consists of considering two plasma rest frames, the upstream frame and the downstream one.
We use indices ' 1 ' or ' 2 ' to indicate quantities measured in the upstream or the downstream frame, respectively. If one neglects the second-order Fermi acceleration, the particle energy is a constant of motion in any of these plasma rest frames and energy changes occur when the particle momentum is Lorentz-transformed at each crossing of the shock. In the case of parallel shock, with the mean magnetic field parallel to the shock normal, the acceleration of an individual particle is due to the consecutive shock crossings by the diffusive wandering particle. Each upstream-downstream-upstream diffusive loop results in a small increment of particle momentum, $\Delta p \propto p \cdot\left(U_{1}-U_{2}\right) / v$, where $v$ is the particle velocity and $U_{i}$ is the shock velocity in the respective $i=1$ or 2 frame, $U_{1} \ll v$. One should note that in oblique shocks, the particle helical trajectory can cross the shock surface a number of times at any individual shock transition or reflection.

The most interesting feature of the first-order Fermi acceleration at a non-relativistic plane-parallel shock wave is the independence of the test-particle stationary particle energy spectrum from the background conditions near the shock, including the mean magnetic field configuration and the spectrum of MHD turbulence. The main reason behind that is a nearly-isotropic form of the particle momentum distribution at the shock. If a sufficient amount of scattering occurs near the shock, this condition always holds for the shock velocity along the upstream magnetic field $U_{B, 1} \equiv U_{1} / \cos \Psi_{1} \ll v\left(\Psi_{1}\right.$ is the upstream magnetic field inclination to the shock normal). Independently of the field inclination at the shock, the particle density is continuous across it and the spectral index for the phase-space distribution function, $\alpha$, is given exclusively in the terms of a single parameter the shock compression ratio $R$ :

$$
\alpha=\frac{3 R}{R-1}
$$




\section{OSTROWSKI}

Because of the isotropic form of the particle distribution function, the spatial diffusion equation has become a widely used mathematical tool for describing particle transport and acceleration processes in non-relativistic flows. With its use the characteristic acceleration time scale at the parallel $\left(\Psi_{1}=0\right)$ shock can be derived as

$$
T_{\mathrm{acc}}=\frac{3}{U_{1}-U_{2}}\left\{\frac{\kappa_{1}}{U_{1}}+\frac{\kappa_{2}}{U_{2}}\right\}
$$

where $\kappa_{i} \equiv \kappa_{\|, i}$ is the respective particle spatial diffusion coefficient along the magnetic field, as discussed by [7]. Ostrowski [8] (see also [9]) derived an analogous expression for shocks with oblique magnetic fields and small amplitude magnetic field perturbations. For a negligible cross-field diffusion and for $U_{B, 1} \ll c$ it can be written in essentially the same form as the one given in Eq. (2.2), with all quantities taken as the normal $(n)$ ones with respect to the shock $\left(\kappa_{n, i}\right.$ for $\left.\kappa_{i}(i=1,2)\right)$. As $\kappa_{n}<\kappa_{\|}$, the oblique shocks may be more rapid accelerators when compared to the parallel shocks.

Not discussed here non-linear and time dependent effects, inclusion of additional energy losses and gains, etc., make the physics of the acceleration more intricate, allowing, e.g. for non-power-low and/or non-stationary particle distributions.

\section{COSMIC RAY ACCELERATION AT RELATIVISTIC SHOCK WAVES}

\section{A. The Fokker-Planck description of the acceleration process}

In the case of the shock velocity (or its projection $U_{B, 1}$ ) reaching values comparable to the light velocity, the particle distribution at the shock becomes anisotropic. This fact complicates to a great extent both the physical picture and the mathematical description of particle acceleration. The first attempt to consider the acceleration process at the relativistic shock was presented in 1981 by Peacock (see also [1]); however, no consistent theory was proposed until a paper of Kirk \& Schneider [11] (see also [12]) appeared. Those authors considered the stationary solutions of the relativistic Fokker-Planck equation for particle pitch-angle diffusion for the case of the parallel shock wave. In the situation with the gyro-phase averaged distribution $f(p, \mu, z)$, which depends only on the unique spatial co-ordinate $z$ along the shock velocity, and with $\mu$ being the pitch-angle cosine, the equation takes the form:

$$
\Gamma(U+v \mu) \frac{\partial f}{\partial z}=C(f)+S
$$

where $\Gamma \equiv 1 / \sqrt{1-U^{2}}$ is the flow Lorentz factor, $C(f)$ is the collision operator and $S$ is the source function. In the presented approach, the spatial co-ordinates are measured in the shock rest frame, while the particle momentum co-ordinates and the collision operator are given in the respective plasma rest frame. For the applied pitchangle diffusion operator, $C=\partial / \partial \mu\left(D_{\mu \mu} \partial f / \partial \mu\right)$, they generalised the diffusive approach to higher order terms in particle distribution anisotropy and constructed general solutions at both sides of the shock which involved solutions of the eigenvalue problem. By matching two solutions at the shock, the spectral index of the resulting power-law particle distribution can be found by taking into account a sufficiently large number of eigenfunctions. The same procedure yields the particle angular distribution and the spatial density distribution. The low-order truncation in this approach corresponds to the standard diffusion approximation and to a somewhat more general method described by Peacock. The above analytic approach (or the 'semi-analytic' one, as the mentioned matching of two series involves numerical fitting of the respective coefficients) was verified by Kirk \& Schneider [13] by the method of particle Monte Carlo simulations.

An application of this approach to more realistic conditions - but still for parallel shocks — was presented by Heavens \& Drury [14], who investigated the fluid dynamics of relativistic shocks (cf. also [15]) and used the results to calculate spectral indices for accelerated particles (Fig. 1). They considered the shock wave propagating into electron-proton or electron-positron plasma, and performed calculations using the analytic method of Kirk \& Schneider for two different power spectra for the scattering MHD waves. In contrast to the non-relativistic case, they found (see also [12]) that the particle spectral index depends on the form of the wave spectrum. The unexpected fact was noted that the non-relativistic expression (2.1) provided a quite reasonable approximation to the actual spectral index.

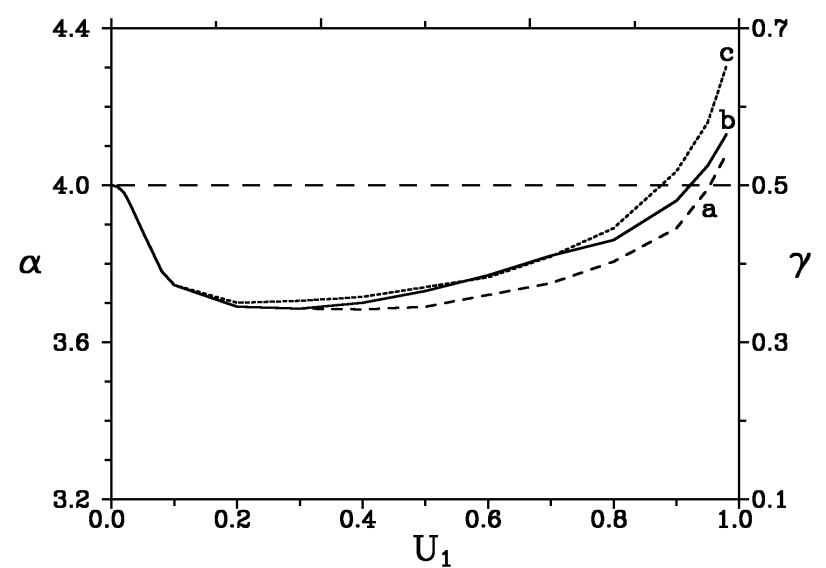

Fig. 1. The particle spectral indices $\alpha$ at parallel shock waves propagating in the cold $(e, p)$ plasma versus the shock velocity $U_{1}[14]$. On the right vertical axis the respective synchrotron spectral index $\gamma$ is given. Using the solid line (b) and the dashed line (a) we show indices for two choices of the turbulence spectrum. The dashed line (c) gives the spectral index derived from Eq. (2.1). The horizontal line $\alpha=4.0$ is given for the reference. 
A substantial progress in understanding the acceleration process in the presence of highly anisotropic particle distributions is due to the work of [16] (see also [17] and [18]), who considered particle acceleration at subluminal $\left(U_{B, 1}<c\right)$ relativistic shocks with oblique magnetic fields. They assumed the magnetic momentum conservation, $p_{\perp}^{2} / B=$ const, at particle interaction with the shock and applied the Fokker-Planck equation discussed above to describe particle transport along the field lines outside the shock, while excluding the possibility of cross-field diffusion. In the cases when $U_{B, 1}$ reached relativistic values, they derived very flat energy spectra with $\gamma \approx 0$ at $U_{B, 1} \approx 1$ (Fig. 2). In such conditions, the particle density in front of the shock can substantially - even by a few orders of magnitude - exceed the downstream density (see the curve denoted ' -8.9 ' at Fig. 3). Creating flat spectra and great density contrasts is due to the effective reflections of anisotropically distributed upstream particles from the region of compressed magnetic field downstream of the shock. However, the conditions leading to very flat spectra are supposed to be accompanied by processes - like a large amplitude wave generation upstream of the shock - leading to spectrum steepening (cf. Sec. III.B).

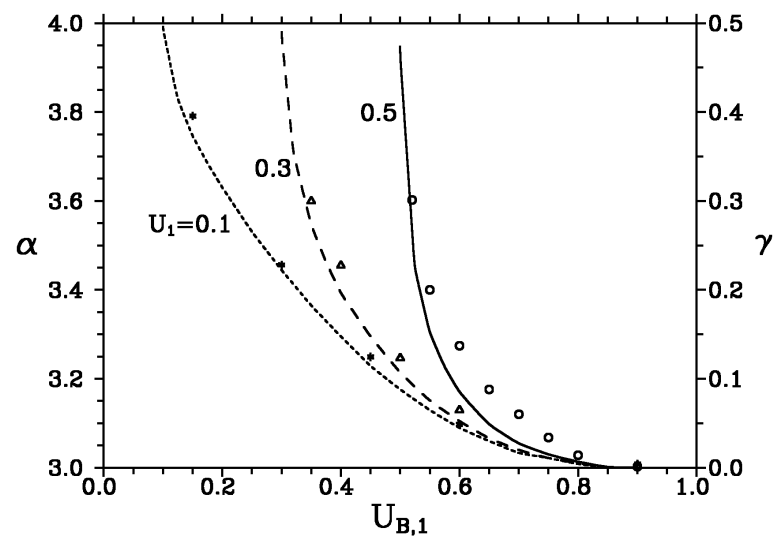

Fig. 2. Spectral indices $\alpha$ of particles accelerated at oblique shocks versus shock velocity projected at the mean magnetic field, $U_{B, 1}$. On the right the respective synchrotron spectral index $\gamma$ is given. The shock velocities $U_{1}$ are given near the respective curves taken from [16]. The points were taken from simulations deriving explicitly the details of particle-shock interactions [8]. The results are presented for compression $R=4$.

As stressed by Begelman \& Kirk [19], in relativistic shocks one can often find the superluminal conditions with $U_{B, 1}>c$, where the above presented approach is no longer valid. Then, it is not possible to reflect upstream particles from the shock and to transmit downstream particles into the upstream region. In effect, only a single transmission of upstream particles re-shapes the original distribution by shifting particle energies to larger values. The energy gains in such a process, involving a highly anisotropic particle distribution, can be quite significant, exceeding the value expected for the adiabatic compression.
The approach proposed by Kirk \& Schneider [11] and [16], and the derivations of [19] are valid only in case of weakly perturbed magnetic fields. However, in the efficiently accelerating shocks one may expect large amplitude waves to be present, when both the Fokker-Planck approach is no longer valid and the magnetic momentum conservation no longer holds for oblique shocks. In such a case, numerical methods have to be used.

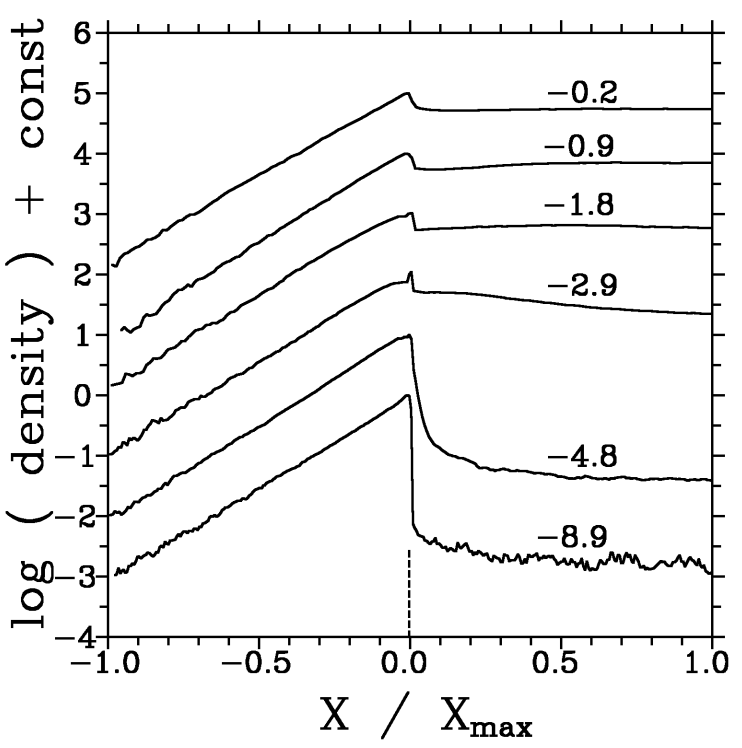

Fig. 3. The energetic particle density across the relativistic shock with an oblique magnetic field [20]. The shock with $U_{1}=0.5, R=5.11$ and $\psi_{1}=55^{\circ}$ is considered. The curves for different perturbation amplitudes are characterized with the value $\log \kappa_{\perp} / \kappa_{\|}$given near the curve. The data are vertically shifted for picture clarity. The value $X_{\max }$ is the distance from the shock at which the upstream particle density decreases to $10^{-3}$ part of the shock value.

\section{B. Particle acceleration in the presence of large amplitude magnetic field perturbations}

The first attempt to consider the acceleration process at parallel shock wave propagating in a turbulent medium was presented by [21], who included into Eq. (3.1) the Boltzmann collision operator describing the large angle scattering. By solving the resulting integrodifferential equation they demonstrated the hardening of the particle spectrum due to increasing contribution of the large-angle scattering. The reason for such a spectral change is the additional isotropization of particles interacting with the shock, leading to an increase in the particle mean energy gain. In oblique shocks, this simplified approach cannot be used because the character of individual particle-shock interaction - reflection and transmission characteristics - depends on the magnetic field perturbations. Let us additionally note that application of the point-like large-angle scattering model in 


\section{OSTROWSKI}

relativistic shocks does not provide a viable physical representation of the scattering at MHD waves [9].

To handle the problem of the particle spectrum in a wide range of background conditions, the Monte Carlo particle simulations were proposed $[8,9,13,22-26]$. At first, let us consider subluminal shocks. The field perturbations influence the acceleration process in various ways. As they enable the particle cross field diffusion, a modification (decrease) of the downstream particle's escape probability may occur. This factor tends to harden the spectrum. Next, the perturbations decrease particle anisotropy, leading to an increase of the mean energy gain of reflected upstream particles, but - what is more important for oblique shocks - this also increases the particle upstream-downstream transmission probability due to less efficient reflections, enabling them to escape from further acceleration. The third factor is due to perturbing particle trajectory during an individual interaction with the shock discontinuity and breakdown of the approximate conservation of $p_{\perp}^{2} / B$. Because reflecting a particle from the shock requires a fine tuning of the particle trajectory with respect to the shock surface, even small amplitude perturbations can decrease the reflection probability in a substantial way. Simulations show (see Fig. 4 for $U_{B, 1}<1.0$ ) that - until the wave amplitude becomes very large - the factors leading to efficient particle escape dominate with the resulting steepening of the spectrum to $\gamma \sim 0.5 \div 0.8$, and the increased downstream transmission probability lowers the cosmic ray density contrast across the shock (Fig. 3).

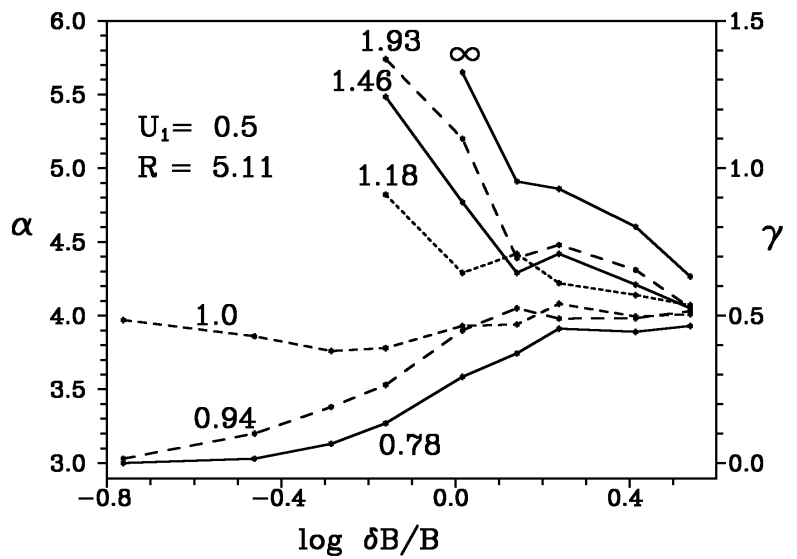

Fig. 4. Spectral indices for oblique relativistic shocks versus perturbation amplitude $\delta B / B$ [23]. Different field inclinations are characterized by the values of $U_{B, 1}$ given near the respective results, $U_{B, 1}<1$ for subluminal shocks and $U_{B, 1} \geq 1$ for superluminal ones. Absence of data for small field amplitudes in superluminal shocks is due to extremely steep power law spectra occurring in these conditions (cf. [18]). Decreasing the field inclination $\Psi_{1} \rightarrow 0$ (i. e., to the parallel shock with $U_{B, 1}=U_{1}$ ) gives spectral indices more and more similar to a constant line $\alpha=3.72$, not shown here for picture clarity (cf. Figs. 1,2).

In parallel shock waves propagating in a highly turbulent medium, the effects discovered for oblique shocks can also manifest their presence because of the local perturbed magnetic field compression at the shock. The problem was considered using the technique of particle simulations by Ballard \& Heavens [24] (cf. [27] for nonrelativistic shock). They showed a possibility of having a very steep spectrum in this case, with the spectral index growing from $\gamma \sim 0.6$ at medium relativistic velocities up to nearly 2.0 at $U_{1}=0.98$. These results apparently do not correspond to the large-perturbation-amplitude limit of [23] (see the discussion therein) simulations for oblique shocks and the analytic results of [14].

For large amplitude magnetic field perturbations the acceleration process in superluminal shocks can lead to the power-law particle spectrum formation, against the statements of [18] valid at small wave amplitudes only. Such a general case was discussed by Ostrowski [23] (see Fig. 4 for $U_{B, 1} \geq 1$ ) and by Bednarz \& Ostrowski $[9,26]$.

\section{The acceleration time scale}

The shock waves propagating with relativistic velocities also raise interesting questions pertaining to the cosmic ray acceleration time scale, $T_{\text {acc. }}$. A simple comparison to non-relativistic values shows that $T_{\text {acc }}$ relatively decreases with increasing shock velocity for parallel [22,28] and oblique [25,29-32] shocks. However, the numerical approaches used there, based on assuming particle isotropization for all scatterings, neglect or underestimate a significant factor affecting the acceleration process - the particle anisotropy. Ellison et al. [22] and Naito \& Takahara [25] also included the more realistic, in our opinion, derivations involving the pitch-angle diffusion approach. The calculations of Ellison et al. for parallel shocks show similar results to those they obtained for large amplitude scattering. For the shock with velocity $0.98 c$ the acceleration time scale is reduced by the factor $\sim 3$ with respect to the non-relativistic formula of Eq. 2.2 . Naito \& Takahara considered shocks with oblique magnetic fields. They confirmed the reduction of the acceleration time scale with an increasing inclination of the magnetic field, derived earlier for nonrelativistic shocks. However, their approach neglected effects of particle cross field diffusion and assumed the adiabatic invariant conservation in particle interactions with the shock, thus limiting the validity of their results to a small amplitude turbulence near the shock.

A wider discussion of the acceleration time scale is presented by Bednarz \& Ostrowski [9], who apply numerical simulations involving the small angle particle momentum scattering. The approach is also believed to provide a reasonable description of particle transport in the presence of large $\delta B$, and thus to enable modelling of the effects of cross-field diffusion. The resulting values (Figs. 5, 6) are given in the shock normal rest frame (cf. [19]). In parallel $\left(\Psi_{1}=1^{\circ}\right)$ shocks $T_{\text {acc }}$ diminishes with the growing perturbation amplitude and shock velocity $U_{1}$. However, it is approximately constant for a given value of $U_{1}$ if we use the formal diffusive time scale, $\kappa_{1} /\left(U_{1} c\right)+\kappa_{2} /\left(U_{2} c\right)$, as the time unit. A new feature discovered in oblique 
shocks is that due to the cross-field diffusion $T_{\text {acc }}$ can change with $\delta B$ in a non-monotonic way (Fig. 5). The acceleration process leading to the power-law spectrum is possible in superluminal shocks only in the presence of large amplitude turbulence. Then, in contrast to the quasi-parallel shocks, $T_{\mathrm{acc}}$ increases with increasing $\delta B$. In the considered cases with the oblique field configurations one may note a possibility to have an extremely short acceleration time scale comparable to the particle gyroperiod in the magnetic field upstream of the shock. A coupling between the acceleration time scale and the particle spectral index is presented in Fig. 6. One should note that the form of involved relation is contingent to a great extent on the magnetic field configuration.

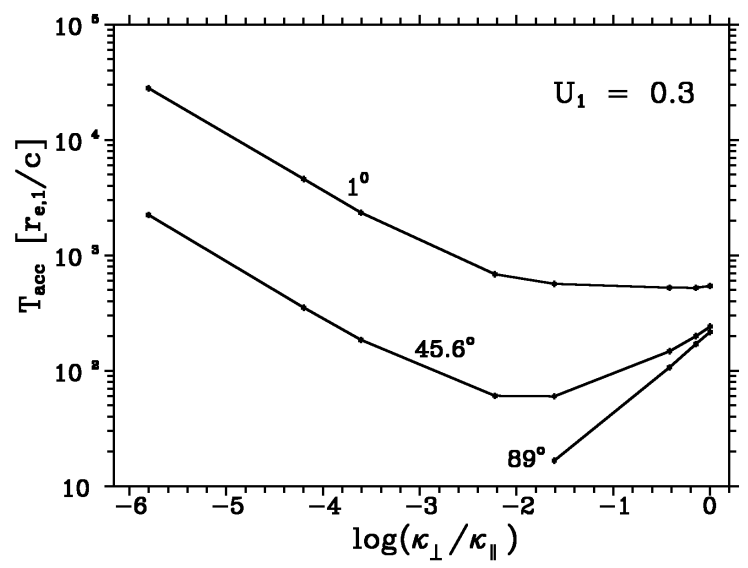

Fig. 5. The acceleration time $T_{\text {acc }}$ versus the level of particle scattering measured by the ratio of $\kappa_{\perp} / \kappa_{\|}$[9]. We present results for three values of the magnetic field inclination: a.) parallel shock $\left(\psi_{1}=1^{\circ}\right)$, b. $)$ a subluminal shock with $\psi_{1}=45.6^{\circ}$ and c.) a superluminal shock with $\psi_{1}=89^{\circ} \cdot r_{e, 1}$ is the particle gyroradius in the effective (including perturbations) upstream magnetic field.

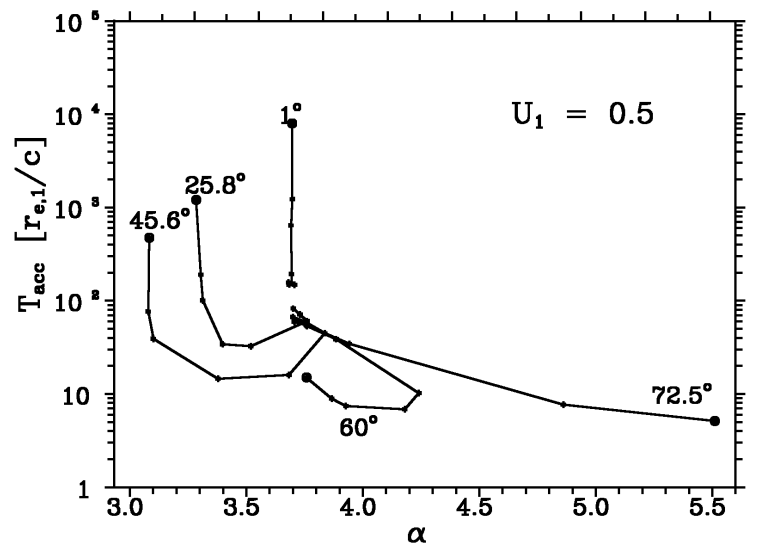

Fig. 6. The relation of $T_{\mathrm{acc}}$ versus the particle spectral index $\alpha$ at different magnetic field inclinations $\psi_{1}$ given near the respective curves. The minimum value of the model parameter $\kappa_{\perp} / \kappa_{\|}$occurs at the encircled point of each curve and the wave amplitude monotonously increases along each curve up to $\delta B \sim B ; r_{e, 1}-$ see Fig. 5 .

\section{ENERGY SPECTRA OF COSMIC RAYS ACCELERATED AT LARGE LORENTZ-FACTOR SHOCKS}

Ultra-relativistic shock waves suggested to be sources of gamma-ray bursts are also expected by some authors to produce ultra-high-energy cosmic ray particles. The process of the first-order Fermi acceleration in such shocks was discussed in a series of papers by Bednarz \& Ostrowski [33,26] (see also [34-38] and [39]). Below, following Ostrowski \& Bednarz [2] we shortly compare and discuss different approaches to the considered acceleration process.

\section{A. The first-order Fermi acceleration at ultra-relativistic shocks}

The first-order Fermi acceleration process at an ultra-relativistic shock wave involves extreme particle anisotropy at the shock in the upstream plasma rest frame (UPF), and more mild distributions in the shock normal rest frame or the downstream plasma rest frame (cf. [19]). Let us consider an individual cosmic ray particle acceleration starting with a particle crossing the shock upstream (cf. [40]). Then, in UPF, its momentum is nearly parallel to the shock normal. When the shock Lorentz factor is large $(\Gamma \gg 1)$ the particle stays in front of the shock for a time required for a slight, $\sim 1 / \Gamma$, deflection of its momentum allowing the shock to overtake it and transmit to the downstream region. The deflection proceeds due to the magnetic field upstream of the shock, consisting of the large scale smooth background structure perturbed by the MHD fluctuations. This tiny change of particle momentum upstream of the shock allows for its transmission downstream of the shock, where - due to the Lorentz transformation with a large $\Gamma$ - its momentum direction can be changed at a large angle with respect to its original direction before the transmission upstream. Such large amplitude angular scatterings can enable a finite fraction of particles to follow trajectories leading to successive transmissions upstream of the shock. Repeating of the described loops, with each roughly doubling the particle energy, leads to formation of the power law particle spectrum. Several authors $[26,36,40]$ discussed this process leading to formation of the spectrum with the energy spectral index $\sigma \approx 2.2$ at $\Gamma \gg 1$. Essentially the same results were obtained within different approaches presented by the above authors and by Kirk et al. [38] and Vietri [39].

The work of $[26,33]$ was based on Monte Carlo simulations of particle transport governed by small amplitude pitch angle scattering. Thus, depending on the mean time between successive scattering acts, $\Delta t$, and the maximum angular scattering amplitude, $\Delta \Omega_{\max }$, it was possible to model situations with different mean field configurations and different amounts of turbulence. The mean field configuration downstream of the shock was 


\section{OSTROWSKI}

derived from the mean upstream field using the appropriate jump conditions and trajectories of particles interacting with the shock discontinuity were derived exactly for such fields. The approach takes into account correlations in the process due to the regular part of the magnetic field, but irregularities responsible for pitch angle scattering are introduced as random. In order to model particle pitch angle diffusion upstream of the shock, with nearly a delta-like angular distribution an extremely small scattering amplitude should be used, $\Delta \Omega_{\max } \ll \Gamma^{-1}$. Increasing the shock Lorentz factor results in decreasing the momentum perturbation required for its transmission downstream and leaves a shorter time for this perturbation, $t_{1}$. In the applied pitch angle diffusion approach the momentum variation due to the regular component of the magnetic field scales like $t_{1}$, whence the diffusive change scales like $t_{1}^{1 / 2}$. Thus growing $\Gamma$ leads to decreasing $t_{1}$ and the diffusive term have to dominate at sufficiently large $\Gamma$. However, one should note that with decreasing $\Delta t$ and $\Delta \Omega_{\max }$, when the interaction proceeds at the subresonance $\left(\ll r_{g}\right)$ spatial scale, a serious physical problem with the applied approach appears: it requires the large amplitude short wave turbulence to be non-linear at shortest scales.

An analogous, pitch angle diffusion modelling appended considerations of [36] (for a more detailed description see [37]), who obtained essentially the same spectral indices as the asymptotic one derived by Bednarz \& Ostrowski [26]. They considered a highly turbulent conditions near the shock leading to the particle pitch angle diffusion with respect to the shock normal, i.e., the regular part of the magnetic field (or continuity of the field across the shock) was neglected. Thus, for example, if the amplitude of the magnetic field turbulence is limited, it can not reproduce spectrum steepening (or flattening at intermediate Lorentz factors) in the presence of oblique magnetic fields (cf. [19,23,26]). The both above models describe essentially the same physical situation only for shocks propagating in the highly turbulent medium.

An alternative discussion of the acceleration process presented by Gallant \& Achterberg [40] was based on a simple turbulence model. In their approach a highly turbulent magnetic field configuration was assumed upstream and downstream of the shock, idealized as cells filled with randomly oriented uniform magnetic fields. With such an approach particles crossing the shock enter a new cell with a randomly selected magnetic field configuration. Thus, there always occur configurations allowing some particles crossing the shock downstream to reach it again and to form the power law spectrum. In this model there is no need for the upstream magnetic field perturbations and a model with the uniform upstream field yields the same power law distribution.

Two quasi-analytic approaches to the considered acceleration process were presented by [38] and Vietri [39]. Both attempt to solve the Fokker-Planck equation describing particle advection with the general plasma flow and the small amplitude scattering of particle pitch angle as measured with respect to the shock normal. The important work of Kirk et al. modified the [11] series expansion approach to treat the delta-like angular distribution upstream of the shock. An analytically more simple Vietri approach applies convenient ansatzes for the anisotropic upstream and downstream particle distributions, resembling the [41] approach to acceleration at 'ordinary' relativistic shocks. Both methods confirm the results of the earlier numerical modelling. A deficiency of the above semi-analytic approaches is its inability to treat situations with mildly perturbed magnetic fields, on average oblique to the shock normal. If considered valid for different magnetic field configurations these models require the large amplitude short wave turbulence to remove any signature of the uniform background field or of the long wave perturbations.

\section{B. Acceleration at the ultra-relativistic shock near the Crab Pulsar}

In the discussion above, to treat the shock as the flow discontinuity, and the acceleration process to be of the first-order Fermi type, one had to consider very high energy particles. Quite interesting alternative approach intended to study the acceleration process starting from low 'thermal' energies was proposed by Hoshino et al. [42] (see also [43]; for review [44]). They considered acceleration at the ultra-relativistic shock formed in the wind outflow of the $\left(\mathrm{e}^{+}, \mathrm{e}^{-}\right)$pair plasma containing heavy nuclei and being permeated by the weak magnetic field oriented perpendicular to the flow direction, i.e., in a model wind for the Crab Pulsar. In the large Lorentz factor wind, the ram pressure of nuclei dominates over the ram pressure of the pair plasma, and both these pressures are much larger than the magnetic field pressure.

At the collisionless shock, the pairs' bulk velocity is isotropized much more efficiently, leaving nuclei penetrating the downstream region as a particle beam. This process generates an electric field in the shock and - due to the ion distribution anisotropy - generates long electromagnetic plasma waves. Damping of such waves by pairs accelerates some of electrons/positrons to energies comparable to the iron nuclei energies downstream of the shock. The work mentioned here is based on the results of numerical plasma simulations of the ultra-relativistic collisionless shock.

\section{FINAL REMARKS}

One may note that observations of possible sites of relativistic shock waves (knots and hot spots in extragalactic radio sources), which allow for the determination of the energetic electron spectra, often yield particle spectral indices close to $\alpha=4.0(\gamma=0.5)$. The theoretical work done to date on the test particle cosmic ray acceleration at mildly relativistic shocks yields not too promising results for meaningful modelling of these astrophysical sources. The main reason for this deficiency is - in contrast to the non-relativistic shocks - a direct 
dependence of the derived spectra on the conditions near the shock. Not only the shock compression ratio, but also other parameters, like the mean inclination of the magnetic field or the turbulence spectrum and its amplitude, are significant. Depending on the actual conditions one may obtain spectral indices as flat as $\alpha=3.0(\gamma=0.0)$ or very steep ones with $\alpha>5.0(\gamma>1.0)$. The background conditions leading to the very flat spectra are probably subject to some instabilities; however, there is no detailed derivation describing the instability growth and the resulting cosmic ray spectrum modification.

The situation was supposed to be simpler for large $\Gamma$ shocks, where the spectral index seems to converge to the universal limit $\sigma_{\infty} \approx 2.2$. However, as pointed out above, the validity of this result may be quite limited. In this moment it is difficult to evaluate if the required conditions are satisfied at the studied ultra-relativistic shocks.

A true progress in modelling particle acceleration in actual sources requires a full plasma non-linear description (see also [45]), including the second-order acceleration processes and a feedback of accelerated particles at the turbulent wave fields near the shock wave, the flow modification caused by the cosmic rays' plasma pre-shock compression and, of course, the appropriate boundary conditions. A simple non-linear approach to the parallel shock case was presented by Baring \& Kirk [46], who found that relativistic shocks could be very efficient accelerators. However, it seems to us that in a more general case it will be very difficult to make any substantial progress in that matter. For very flat particle spectra the non-linear acceleration picture depends to a large extent on the detailed knowledge of the background and boundary conditions in the scales relevant for particles near the upper energy cut-off. The existence of stationary solutions is doubtful in this case. A noticeable progress in considering detailed physics of the acceleration in relativistic collisionless shocks may result from application of the particle-in-cell simulations, including physical processes (instabilities) discussed by, e.g., Hoshino et al. [42], Medvedev \& Loeb [47] or Pohl et al. [48].

The present work was supported by the Komitet Badań Naukowych through the grant PB 258/P03/99/17.
[1] M. Ostrowski, in Frontier Objects in Astrophysics and Particle Physics (Vulcano Workshop), edited by F. Giovannelli, G. Mannocchi (1999), p. 319.

[2] M. Ostrowski, J. Bednarz, (2002), submitted.

[3] L. O' C. Drury, Rep. Prog. Phys. 46, 973 (1983).

[4] R. D. Blandford, D. Eichler, Phys. Rep. 154, 1 (1987).

[5] E. G. Berezhko, W. K. Iolshin, G. F. Krymskij, S. I. Pietukhov, Cosmic Ray Generation at Shock Waves (Nauka, Moscow, 1988).

[6] F. C. Jones, D. C. Ellison, Space Sci. Rev. 58, 259 (1991).

[7] P. O. Lagage, C. Cesarsky, Astron. Astrophys. 125, 249 (1983).

[8] M. Ostrowski, Mon. Not. R. Astron. Soc. 233, 257 (1988).

[9] J. Bednarz, M. Ostrowski, Mon. Not. R. Astron. Soc. 283, 447 (1996).

[10] G. M. Webb, Astrophys. J. 296, 319 (1985).

[11] J. G. Kirk, P. Schneider, Astrophys. J. 315, 425 (1987).

[12] J. G. Kirk, Habilitation Theses, preprint No. 345, MaxPlanck-Institut für Astrophysik, Garching, 1988.

[13] J. G. Kirk, P. Schneider, Astrophys. J. 322, 256 (1987).

[14] A. Heavens, L' O. C. Drury, Mon. Not. R. Astron. Soc. 235, 997 (1988).

[15] D. C. Ellison, S. P. Reynolds, Astrophys. J. 382, 242 (1991).

[16] J. G. Kirk, A. Heavens, Mon. Not. R. Astron. Soc. 239, 995 (1989).

[17] M. Ostrowski, Mon. Not. R. Astron. Soc. 249, 551 (1991).

[18] K. R. Ballard, A. F. Heavens, Mon. Not. R. Astron. Soc. 251, 438 (1991).

[19] M. C. Begelman, J. G. Kirk, Astrophys. J. 353, 66 (1990).

[20] M. Ostrowski, in Relativistic Hadrons in Cosmic Compact Objects, edited by A. Zdziarski, M. Sikora (1991), p. 121.
[21] J. G. Kirk, P. Schneider, Astron. Astrophys. 201, 177 (1988).

[22] D. C. Ellison, F. C. Jones, S. P. Reynolds, Astrophys. J. 360, $702(1990)$.

[23] M. Ostrowski, Mon. Not. R. Astron. Soc. 264, 248 (1993).

[24] K. R. Ballard, A. F. Heavens, Mon. Not. R. Astron. Soc. 259, 89 (1992).

[25] T. Naito, F. Takahara, Mon. Not. R. Astron. Soc. 275, 1077 (1995).

[26] J. Bednarz, M. Ostrowski, Phys. Rev. Lett. 80, 3911 (1998).

[27] M. Ostrowski, Astron. Astrophys. 206, 169 (1988).

[28] J. J. Quenby, R. Lieu, Nature 342, 654 (1989).

[29] F. Takahara, T. Terasawa, in Proc. ICRR Int. Symp. Astrophysical Aspects of the Most Energetic Cosmic Rays, edited by M. Nagano \& F. Takahara, Kofu (1990).

[30] P. L. Newman, X. Moussas, J. J. Quenby, J. F. ValdesGalicia, Z. Theodossiou-Ekaterinidi, Astron. Astrophys. 255, 443 (1992).

[31] R. Lieu, J. J. Quenby, B. Drolias, K. Naidu, Astrophys. J. 421, 211 (1994).

[32] J. J. Quenby, B. Drolias, in Proc. 24th Int. Cosmic Ray Conf., 3, 261 (Rome, 1995).

[33] J. Bednarz, M. Ostrowski, in Proc. 25th Int. Cosmic Ray Conf. (Durban, OG 9.1.6, 1997).

[34] J. Bednarz, Ph. D. Thesis, Jagiellonian University (astroph/0005075) (2000).

[35] J. Bednarz, Mon. Not. R. Astron. Soc. 315, L37 (2000).

[36] Y. A. Gallant, A. Achterberg, J. G. Kirk, Astron. Astrophys. Supp., 138, 549 (1999).

[37] A. Achterberg, Y. A. Gallant, J. G. Kirk, A. W. Guthmann, Mon. Not. R. Astron. Soc. 328, 393 (2001).

[38] J. G. Kirk, A. W. Guthmann, Y. A. Gallant, A. Achterberg, Astrophys. J. 542, 235 (2000).

[39] M. Vietri, e-print astro-ph/0002269 (2000). 


\title{
M. OSTROWSKI
}

[40] Y. A. Gallant, A. Achterberg, Mon. Not. R. Astron. Soc. 305, L6 (1999).

[41] J. A. Peacock, Mon. Not. R. Astron. Soc. 196, 135 (1981).

[42] M. Hoshino, J. Arons, Y. A. Gallant, A. B. Langdon, Astrophys. J. 390, 454 (1992).

[43] Y. A. Gallant, J. Arons, Astrophys. J. 435, 230 (1994).
[44] J. Arons, Astron. Astrophys. Suppl. Ser. 120, 49 (1996).

[45] M. Ostrowski, Comments Astrophys. 17, 207 (1994).

[46] M. G. Baring, J. G. Kirk, Astron. Astrophys. 241, 329 (1990)

[47] M. V. Medvedev, A. Loeb, Astrophys. J. 526, 697 (1999).

[48] M. Pohl, I. Lerche, R. Schlickeiser, Astron. Astrophys. 383, 829 (2002).

\section{ПРИСКОРЕННЯ КОСМІЧНИХ ПРОМЕНІВ ПРИ РЕЛЯТИВІСТСЬКИХ УДАРАХ}

\author{
М. Островський \\ Астрономічна обсерваторія ЯТеллонсъкого університету \\ вул. Орла, 171, 30-244, Краків, Польща \\ e-mail:mio@oa.uj.edu.pl
}

\begin{abstract}
Зроблено огляд теоретичних дослі,жень прискорення частинок космічних променів у першому порядку фермі-процесу на фронті релятивістських ударних хвиль. Спочатку обговорено процес прискорення, який діє в помірних релятивістських ударних хвилях. Проаналізовано суттєву роль непрямої зміни конфігурації поля в утворенні спектра енергїі частинок і зміни часової шкали прискорення. Далі розглянуто прискорення в ультрарелятивістських ударних хвилях, які часто дають асимптотичний спектральний індекс $\sigma \approx 2.2$ для великих лоренц-факторів. Пояснено, чому цей результат обмежений випадком високотурбулентних умов поблизу фронтів ударних хвиль. Зроблено висновок, шо сучасне розуміння процесів прискорення, які діють у релятивістських ударних хвилях, $є$ недостатнім для реалістичного моделювання ударних хвиль.

Цей огляд є модифікованою, переробленою й модернізованою версією огляду [M. Ostrowski, in Frontier Objects in Astrophysics and Particle Physics (Vulcano Workshop), edited by F. Giovannelli, G. Mannocchi (1999), p. 319.].
\end{abstract}

